\title{
Displaying detail in bright environments: A 10,000 nit display and its evaluation
}

\author{
Jonathan Hatchett ${ }^{\mathrm{a}}$, Domenico Toffoli ${ }^{\mathrm{b}}$, Miguel Melo ${ }^{\mathrm{c}}$, Maximino Bessa ${ }^{\mathrm{c}, \mathrm{d}}$, Kurt Debattista ${ }^{\mathrm{a}}$, \\ Alan Chalmers ${ }^{\text {a,* }}$ \\ ${ }^{a}$ University of Warwick, Coventry, UK \\ b SIM2 BV s.r.l., Italy \\ ${ }^{\mathrm{c}}$ INESC TEC, Porto, Portugal \\ ${ }^{\mathrm{d}}$ Universidade de Trás-os-Montes e Alto Douro, Vila Real, Portugal
}

\section{A B S T R A C T}

Consumer High Dynamic Range (HDR) displays are appearing on the market. Capable of generating a peak luminance of up to 2,000 nits, the improved dynamic range they provide can only be perceived when viewed in a dark environment. In this paper, we present a display architecture that is capable of generating a peak luminance of 10,000 nits. We demonstrate, with a subjective evaluation, that the increased peak luminance is required to perceive a high dynamic range in bright ambient environments. Furthermore, we show that by fitting a surface through the data, we can predict the dynamic range that can be perceived from the luminance and illuminance with low error. We can also invert the prediction to estimate the required peak luminance for a particular combination of dynamic range and ambient lighting.

\section{Introduction}

High Dynamic Range (HDR) imaging has the potential to capture, transmit, and display the full range of light in a scene [1]. The last 15 years has seen HDR video developed from a niche research area to a consumer product [2]. Despite the interest, commercially available display technology requires HDR content be viewed in a dark room [3]. It is not possible to perceive and appreciate the dynamic range in many common ambient environments. This is because the dynamic range in consumer HDR displays, especially those based on organic light-emitting diode (OLED) technology, is achieved due to the deeper black levels rather than the limited peak luminance. Typical consumer backlit liquid-crystal display (LCD) displays have peak luminances of $\leq$ 2000 nit while OLED displays have a peak luminance of $\approx 750$ nit [4] ( $1 \mathrm{nit}=1 \mathrm{~cd} / \mathrm{m}^{2}$, the SI unit of luminance). There have been a number of recent successes in achieving HDR imaging through projection [5], and other significant advances in display technology $[6,7]$. To overcome the limitation of only being able to appreciate HDR in a darkened room, it is crucial to develop and evaluate new, brighter displays.

This paper focuses on the dynamic range that can be recreated by HDR monitors and presents an innovative display which includes a novel heat dissipation method, that is capable of presenting a luminance of 10,000 nits. This monitor was the first capable of emitting such a luminance when it was first exhibited to the public at IBC in September 2016 [8]. Our system exploits a number of techniques to achieve such a peak luminance. (1) a backlight unit containing 2202 LEDs, (2) the high $(150 \mathrm{~lm}$ ) luminous flow of the individual white LEDs in a Surface Mount Technology (SMT) case, (3) a hexagonal shaped reflector to concentrate and direct the luminous flow generated by each LED through the LCD front panel, and (4) an original thermal management system designed to spread the heat generated evenly over the sink mass.

Furthermore, we present a detailed subjective evaluation conducted with 40 participants showing the effects of peak luminance on perceived dynamic range in different ambient lighting conditions. This evaluation demonstrates that a high peak luminance is required for displaying HDR in a bright ambient environment. The results of this evaluation are used to develop a model which allows the prediction of the required peak luminance from the illuminance of the environment and the dynamic range of the content. One benefit to managing the peak luminance of the display in such a way is to ensure an enhanced viewing experience for the minimum power consumption.

\section{Previous work}

The world is a riot of colour, light, detail and motion; from the flicker of flame, to the intensity of neon lights. For years digital imaging technologies have struggled to capture and deliver this vibrant "real world". Ultra High Definition (UHD) is the Creative and Media Industries vision for the future of digital imaging. Formalised in the BT.2020

\footnotetext{
No author associated with this paper has disclosed any potential or pertinent conflicts which may be perceived to have impending conflict with this work. For full disclosure statements refer to https://doi.org/10.1016/j.image.2019.04.011.

* Corresponding author.

E-mail addresses: j.p.hatchett@warwick.ac.uk (J. Hatchett), alan.chalmers@warwick.ac.uk (A. Chalmers).
} 


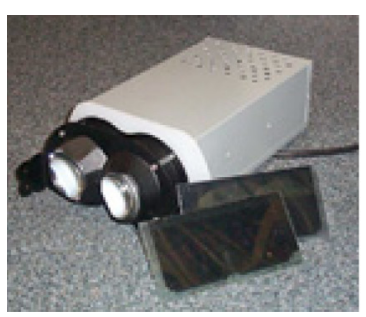

(a) Stereo viewer.

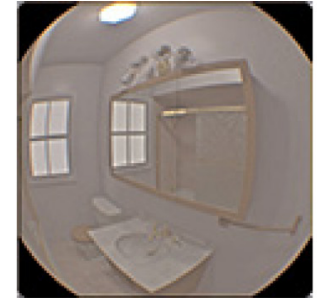

(b) Front transparency.

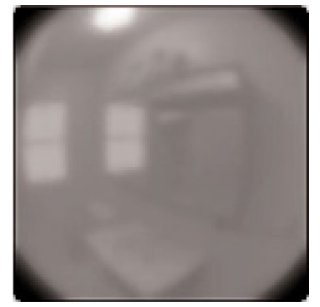

(c) Back transparency.

Fig. 1. Early HDR display [9].

standard [10], UHD specifies that imaging of the future should include more spatial resolution (at least $4 \mathrm{~K}=3840 \times 2160$ pixels), higher dynamic range (HDR), higher frame rate (HFR) and a wider colour gamut (WCG). The dynamic range of a scene is defined as the ratio between the darkest and brightest parts of that scene. By preserving all the details simultaneously in both the darkest and brightest areas of a picture, colours and details look more natural, giving the picture a more "dynamic" feel.

\subsection{HDR display systems}

In 2002, Ward produced arguably the world's first HDR display. This comprised a bright uniform light behind a set of LEEP ARV-1 stereo optics [11]. HDR images were printed side-by-side as a pair of fisheye transparencies. As film transparencies in 2002 were only capable of achieving a dynamic range of $1000: 1$, two transparencies were layered on top of each other. This doubled, in log terms, the dynamic range, achieving more than 10,000:1 [9]. To avoid any alignment or ghosting issues, the resolution of the back layer was reduced using a Gaussian filter, and, as dynamic range of the individual colour channels is not important for perception, the image was converted to grey scale, Fig. 1. A close match in terms of visibility and contrast, was shown between the displayed images and real scenes [12].

Sunnybrook Technologies were the first to develop an HDR display using a backlit standard LCD display. By removing the colour wheel of an Optoma EzPro737 digital light projector and projecting video through the back of a 15" XGA colour Sharp LQ150X1DG0 LCD display, the Sunnybrook Technologies' display was able to achieve a dynamic range of 75,000:1 [13]. Sunnybrook Technologies became Brightside in March 2005 and the next generation of their displays, the DR37$\mathrm{P}$ replaced the projector with LEDs. By combining software correction algorithm that took into account how the human eye perceives high contrast with each LED covering approximately $40 \times 40$ pixels, the liquid cooled prototype DR37-P display achieved a peak luminance of 8500 nit [14]. With the acquisition of Brightside by Dolby Laboratories in February 2007, further developments on HDR displays were undertaken by the Italian company, SIM2. Their first commercial HDR display, the HDR47, was released in 2009 with a peak luminance of 4000 nit. The $47^{\prime \prime}$ HDR47ES6MB HDR display, announced by SIM2 in 2014 has a peak luminance of 6000 nit. Other prototype HDR displays have been built, for example Dolby Laboratories' 4000 nit Pulsar display [15] and by Ferweda and Luca for vision research [16].

Currently, the field of larger format HDR displays (from $\approx 32$ to 75 inch) can be separated into research, professional and consumer displays. In this paper we describe the architecture and innovation required to achieve a 10,000 nit HDR display.

\subsection{Modern consumer HDR displays}

From Standard Definition, to High Definition (HD), to 3D, the media industry continually strives to move forward and provide the next compelling reason why customers must acquire the next generation of products. Driven by the need to sell more televisions, and the lack of very highly appreciable difference on consumers' viewing experience between full HD resolution images and UHD resolution, especially on screens of less than $60^{\prime \prime}$ [17], the television industry looked to other aspects in the UHD specification to excite consumers. As a result, in the last few years, consumer televisions have been appearing which claim to be HDR. In 2016 a consortium of TV manufacturers, broadcasters and content producers, the UHD Alliance, proposed a benchmark, "Ultra HD Premium". Any television which is able to achieve this benchmark is certified by the UHD Alliance as providing a "premium 4K experience". To satisfy manufacturers of both backlit LCD and OLED HDR displays UHD Alliance offers two definitions of HDR:

1. 1000 nit peak brightness and $<0.05$ nit black level (contrast ratio 20,000:1, 14.3 stops) for backlit LCD TVs which are brighter but with less black levels, or

2. $>540$ nit brightness and $<0.000,5$ nit black level (contrast ratio $1,080,000: 1, \approx 20$ stops) for OLED TVs which have deep blacks but much lower peak brightness.

As of 2018, the brightest OLED displays have a peak luminance of $<$ 750 nit while backlit consumer HDR displays provide a peak luminance of $\leq 2000$ nit [18].

A major limitation with current OLED HDR displays, is the need to watch content in a dark room to discern the dynamic range in a scene [19]. For consumer HDR backlit LCD displays, to appreciate the HDR, the recommendation is that the displays should not be watched in ambient light of more than 5 nits [19]. Furthermore, because the maximum backlight brightness is used to provide bright highlights, it is not possible to increase the overall brightness of the screen to get a brighter image as one would with a traditional low dynamic range (LDR) television [20]. If HDR content is to be appreciated in bright ambient conditions, a higher peak luminance is needed.

\section{Architecture for 10,000 nit display}

The architecture for the 10,000 nit display accepts either an LDR or HDR video input source, via HDMI or DVI, and processes this video signal within a digital signal processing block to create HDR images in real-time see Fig. 3. The processing block consists of a regional LED backlight control signal processing block and a complimentary "expanded" and compensated LCD video signal processing block, that, when combined in a panel display system, provide the HDR viewing experience. The resolution of the LCD panel is full HD. Although the transmissivity is higher for a full HD LCD panel compared to that of a UHD LCD panel, the improvements made to the passage of light in this 10,000 nit display makes this difference insignificant.

The input signal can be either 8,10 or 12 bits/colour. This is expanded into a 30 bits/colour HDR signal. This colour depth is achieved from a combination of the BLU and the LCD bit depth via a double modulation system.

The HDR processing module, see Fig. 2, calculates the LED values and the required compensation for the LCD image and is responsible for driving the backlight boards at a frame rate of $60 \mathrm{~Hz}$. The HDR input format is LogLUV, the display does not support PQ or HLG natively. 


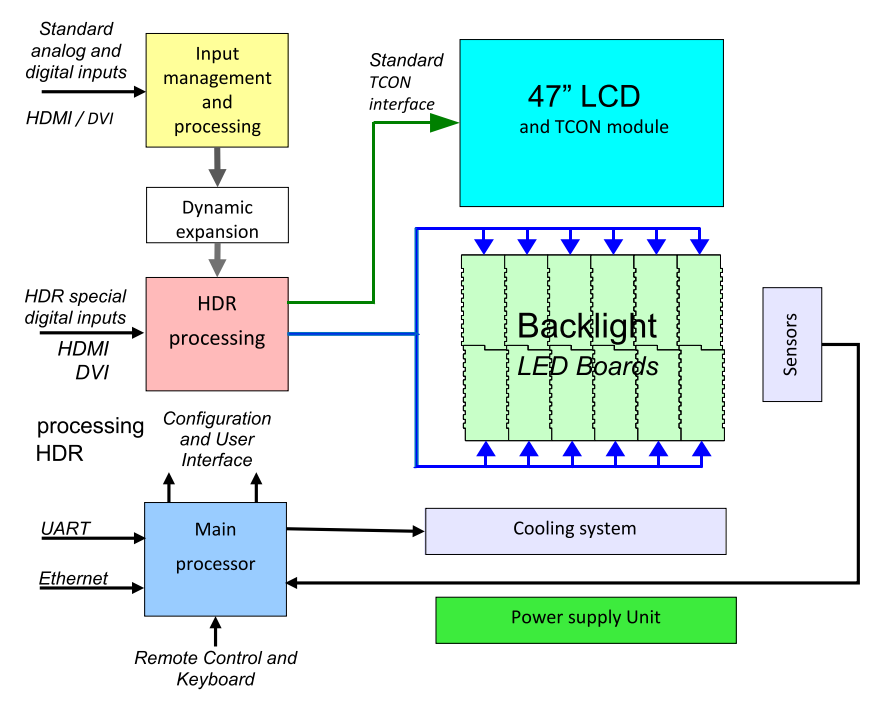

Fig. 2. The physical architecture of the 10,000 nit display.

The backlight unit performs the calculation at $60 \mathrm{~Hz}$ frame rate. The monitor diagonal is $47^{\prime \prime}$ with a $16: 9$ aspect ratio, with an active LCD area of $0.62 \mathrm{~m}^{2}$. The LCD refresh frequency is higher and is independent from frame frequency. A main processor provides user and control interfaces, on-screen display, and system configuration.

The TFT front-panel contains Rec.709 [21] primary colour filters limiting the display colour-space.

\subsection{The backlight unit}

In consumer LCD TV sets and monitors, there are several optical layers between the backlight source and the LCD panel. These include the diffuser and the two polarising layers. The majority of luminance generated by the backlight unit (BLU) is lost passing through these optical layers. In addition, luminance is lost due to the TFT array aperture ratio and panel colour filters of the LCD panel itself. The result is that only 3 to $8 \%$ of the light emitted by the BLU actually exits the front of the screen.

The core of the 10,000 nit display is a BLU comprising an array of 2202 independently controlled high power white LEDs. A luminance of 10,000 nit corresponds to a light intensity of 6200 candela (cd) and a luminous flow of 19,500 lumens $(\mathrm{lm})$. Taking into consideration the LCD panel efficiency, this means that, to achieve 10,000 nit, the BLU has to provide a total luminous flow of at least $330,000 \mathrm{~lm}$. The BLU thus comprises the 2202 power white LEDs in SMT cases arranged in a hexagonal grid. High quality LEDs are used, each capable of $150 \mathrm{~lm}$. The LEDs are all selected from the same bin and production lot to minimise any differences between them which can lead to an uneven brightness. As LEDs are Lambertian light emitters, in order to concentrate and direct the luminous flow generated by each LED in the direction of the LCD panel, reflectors are added for each LED. These reflectors reduce the light energy in the tails of the LED emission and re-direct this energy forward around the centre of the LED reflectors. The reflectors make up a grid which has been optimised for the highest efficiency. A hexagonal shape was shown to be the best and, furthermore, the hexagonal shape minimises light leaking from any LED. The reflector grid is produced with moulded Acrylonitrile Butadiene Styrene (ABS), a high impact-resistance thermoplastic. The distance between the LED reflector grid and the optical layers, the reflective surface type of the reflectors, and the diffusing capability of the panel optical layer need to be as small as possible to prevent a pattern of non-uniform brightness being visible within the image. Each LED module comprises 12 tiles of LED boards that can be assembled easily and combined together to form the full backlight unit.

Each LED emission level is controlled, consistently with the image on the LCD panel and with the specific Light Spreading Function (LSF) of the optical layers, in order to give the most appropriate brightness contribution related to the part of the image assigned to it. In particular, the LED module receives a serialised Low-Voltage Differential Signalling (LVDS) signal from the HDR processing module. This LVDS signal is deserialised and the converted signal fed to the LED driver IC chain that provides a separate driving signal to each LED. Sequencing and synchronisation is required to coincide with the scanning of the corresponding section of the LCD display, Fig. 4. The approach is similar to, but more effective, that undertaken in [13]. In addition, optical and thermal sensors provide feedback to the HDR micro processing in order to maintain reliability and system optical uniformity over space and time. This is particularly important because the light and spectral emissions of LEDs are a function of the working temperature. Furthermore, a LEDs lifetime is reduced with high temperatures.

\subsection{Thermal considerations}

A key requirement to help ensure the widespread uptake of HDR displays is to safeguard that very little heat is generated and the power consumed by a display is within that which customers are prepared to tolerate [22]. A high current of up to $300 \mathrm{~mA}$ with $4 \mathrm{~V}$ of voltage drop per LED, leads to a peak power required from the power supply of more than $2600 \mathrm{~W}$. This is more than the maximum power absorption, $1500 \mathrm{~W}$, from a $110 \mathrm{~V} \mathrm{AC}$ power socket in the case of single phase power distribution, and thus the power supply needs to be limited. This reduces the peak full-screen luminance to 3830 nits. The display can

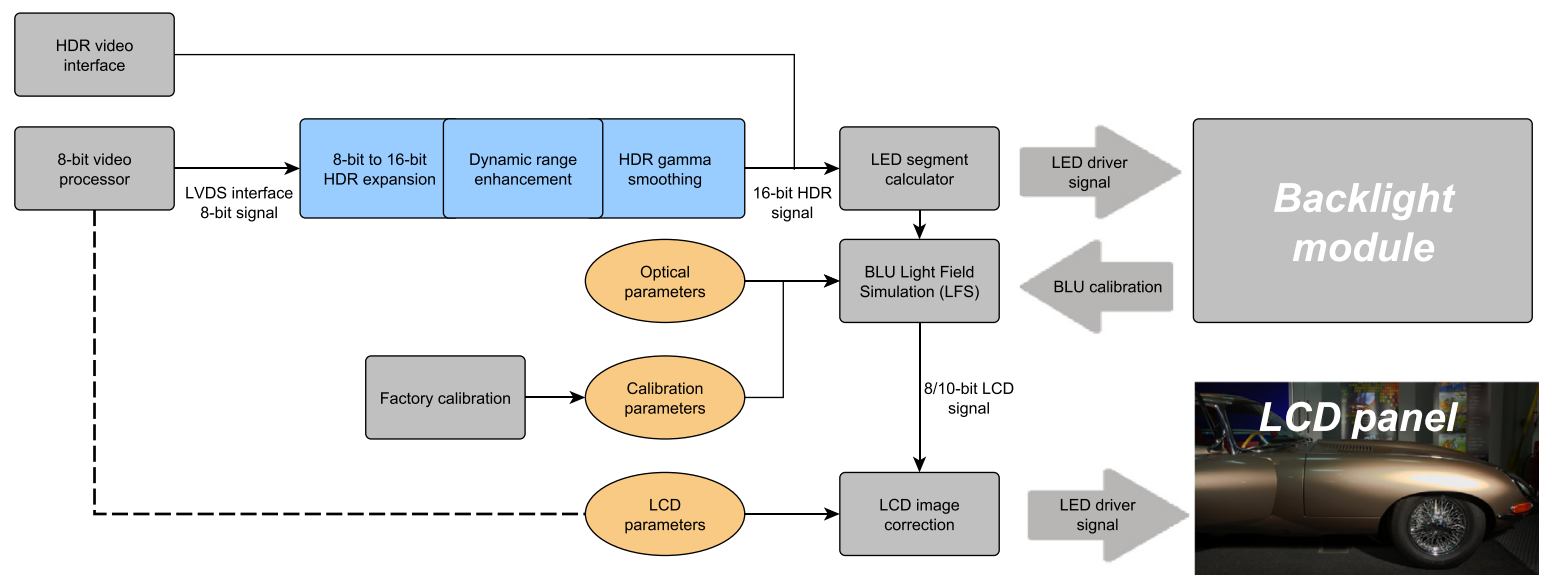

Fig. 3. High level overview of the 10,000 nit display. 


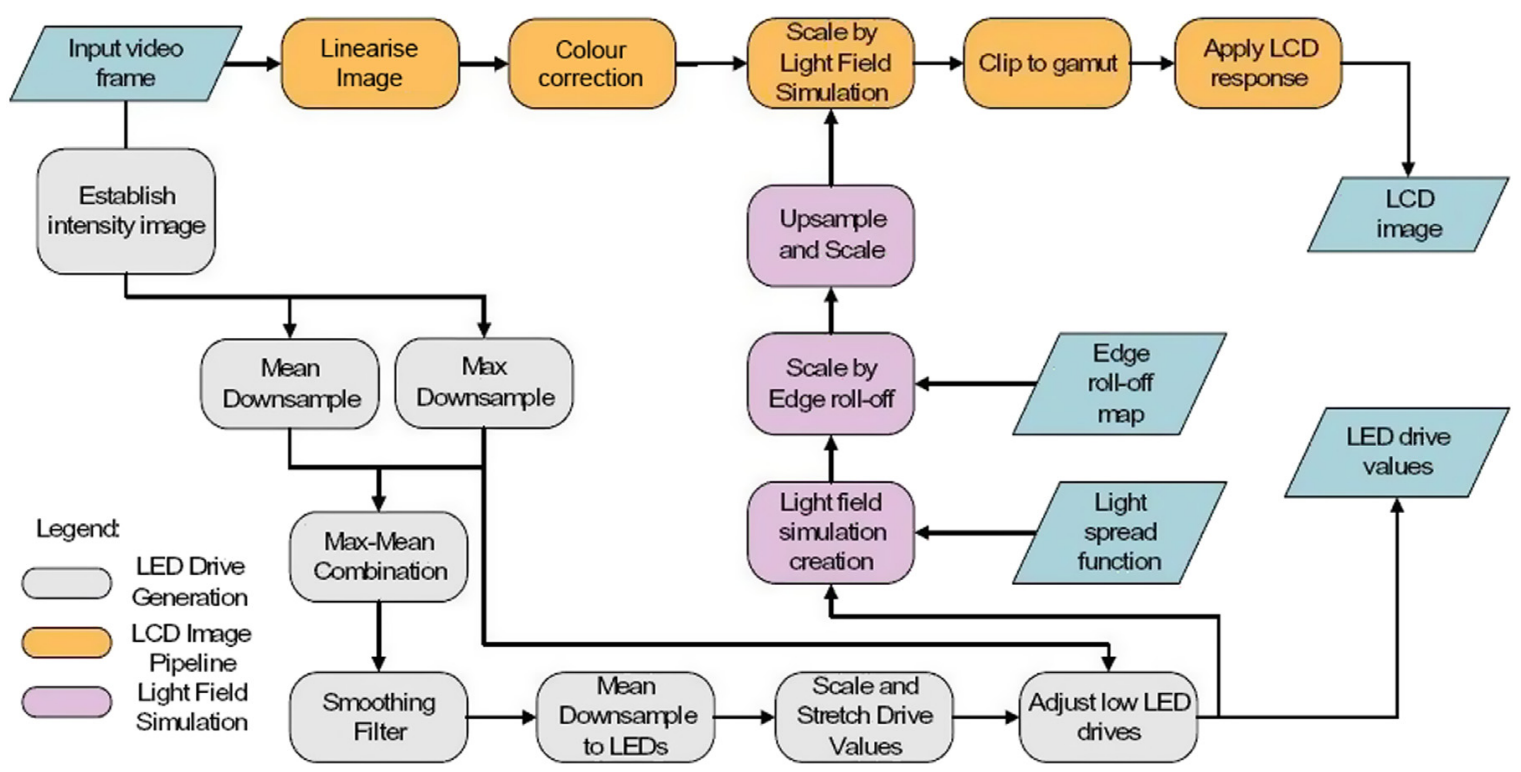

Fig. 4. The processing required to split the HDR image to low and high frequency components for the LED and LCD panels respectively.

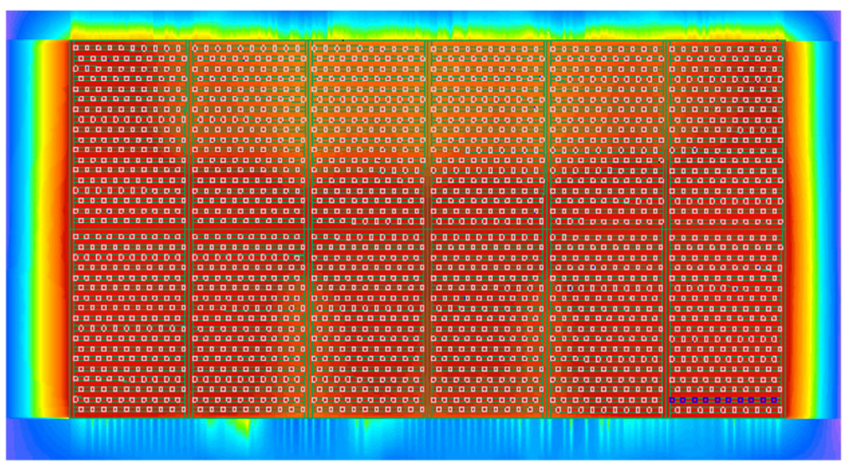

Fig. 5. The measured uniform spatial heat distribution achieved by the novel thermal management system.

output 10,000 nits from a maximum of around $55 \%$ of the total screen area before the power limitation system intervenes.

An effective and efficient cooling system is a key part of any high brightness display. The novel thermal management system has been design to spread the entire heat quantity evenly across the whole heat sink mass, ensuring homogeneity, Fig. 5. The LED modules are mounted with intimate thermal connection to a single aluminium cooling substrate. A set of low RPM, high-efficiency fans force air to travel against the temperature gradient. This configuration increases heat dissipation while helping its distribution. The combination of heat reducing approaches enables the display to achieve cooling performance equivalent to that which would be possible with liquid cooling.

\section{Subjective evaluation}

Many of the environments in which HDR content will be consumed contain bright ambient illuminations. Examples include a television situated close to a window, where, when the content is consumed, the sun may be low in the sky, casting rays directly on to the screen; or an outdoor advertising screen or a public television. This section will demonstrate how the ambient environment affects the perceived detail visible to the subject.

Akyüz et al. [23] demonstrated that participants prefer a HDR image over tone-mapped or LDR image of a scene, Mukherjee et al. [24] showed that this also holds true for HDR video. Melo et al. [25] investigated tone-mapped HDR content on an LDR display to find that the ambient illumination, and especially reflections on the screen, significantly affected the viewing experience. An experiment in which participants watched HDR video on an HDR display under different ambient illuminations showed that visual fatigue was not a particular problem [26]. There was, however, a significant difference in the choice of brightness and contrast depending on the lighting conditions. The two experiments were conducted on a display with a peak luminance of 4000 nit and a black level of $<0.001$ nit at seven ambient illuminations. $<0.01,0.75,8.5,28$ and 74 lux in the first and, $<0.01,70$ and 700 lux in the second. Daly et al. [27] conducted an experiment in a dark room that showed for imagery containing diffuse, reflective whites, the average preferred range of display was between 0.1 to 650 nit. However to satisfy $90 \%$ of the population the diffuse range should be between 0.005 to 3000 nit and greater than 20,000 nit for highlights [28].

\subsection{Motivation}

There have been no studies presented to date that investigate the combination of both high peak luminance displays and bright ambient environments. Peak brightnesses of up to 20,000 nit [27] and ambient illuminations of up to 700 lux [26] have been tested but never in combination and not in environments such as those found outdoors where the ambient illumination can greatly exceed those previously tested.

This section presents an experiment in which both a high peak brightness and a high ambient illumination are tested. The experiment was performed on a prototype SIM2 display capable of producing 10,000 nit, five times that of consumer displays, and in a range of environments up to 80,000 lux. The aim of this experiment is to measure the dynamic range that can be perceived by a participant under a range of real-world ambient illuminations and display brightnesses.

The hypothesis is that in conditions such as outdoors on a sunny day, where the illumination can exceed 80,000 lux [29], a higher peak luminance display will allow more information in the image to be perceived. Fig. 6 shows the relative difference in brightness between a 10,000 nit display and a 4000 nit display in a bright ambient environment. A secondary goal is to be able to calculate the required luminance of a display for content of a known range at a given ambient illumination. This will be achieved by performing a subjective test where participants are asked to report the visibility of elements on a test chart at a range of display luminances and ambient illuminations. 


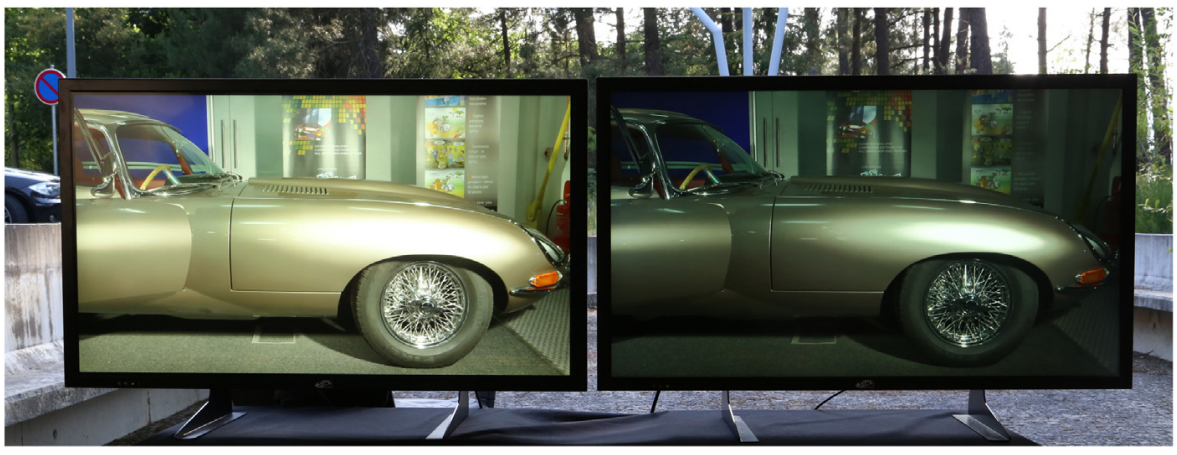

Fig. 6. The 10,000 nit SIM2 alongside the 4000 nit SIM2 in a bright ambient environment.

Table 1

The ambient illuminance of locations for some target applications for HDR displays.

\begin{tabular}{ll}
\hline Application & $\begin{array}{l}\text { Typical ambient illumination } \\
\text { (lux) }\end{array}$ \\
\hline $\begin{array}{l}\text { Home theatre [30] } \\
\text { Advised home environment viewing conditions }\end{array}$ & 200 \\
[30] & 500 \\
Typical office lighting & 3000 \\
Inside on a sunny day & 10,000 \\
Advertising display in shopping centre & 80,000 \\
Hotel pool bar &
\end{tabular}

\subsection{Experiment}

This section presents an experiment which explores the relationship between the detail visible on a display, peak luminance and ambient illumination. Environments covering a large range of lighting conditions were considered and in particular the wide variety of locations in which HDR displays could be employed in the future were simulated. Table 1 shows a selection of typical ambient illuminations at potential locations for HDR displays. Should HDR displays become ubiquitous, it can be expected that they will find application in all environments, such as an advertising display in a shopping centre and at the bar next to a hotel pool.

\subsubsection{Participants}

The sample consisted of 40 participants (27 male and 13 female) with ages between 18 and 49 years old $(\mu=23.75, \sigma=7.0)$ who volunteered from a local university. The participants were divided into eight groups of five each. The experiment used small groups to increase the sample size while care was taken to ensure that every participant was within the stipulated viewing angle for the display. Participants were chosen based on a non-probabilistic sampling technique as they were chosen based on their availability to sit the experiment. Due the nature of the experiment, only participants with normal or corrected to normal vision were chosen. The participants were selected primarily from staff and students at a computer science department however did not express an increased knowledge of image processing or HDR techniques.

\subsubsection{Design}

The experiment comprised three tasks designed to determine the range visible on displays at simulated luminances. These tasks are as follows:

Squares: Participants are asked to count the number of squares, shown in Fig. 7a;

Ayres: $\quad$ Participants are asked to give the orientation of the circles on Ayres charts [31], shown in Fig. 7b.

Bars: $\quad$ Participants are asked to identify the minimum and maximum bars they can discern, shown in Fig. 7c;

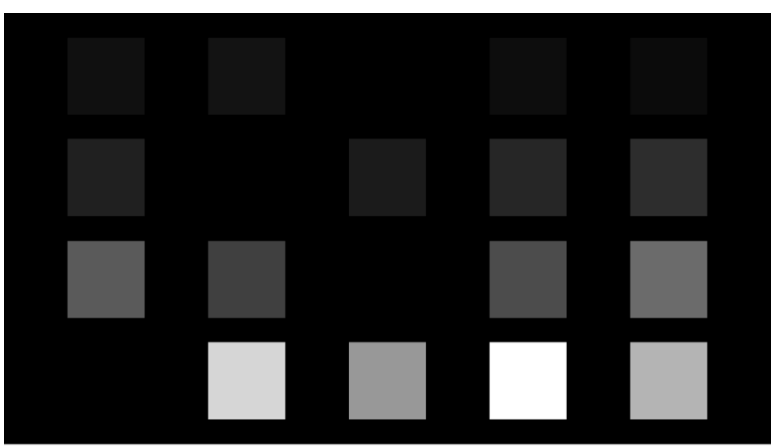

(a) Squares: patches separated by 1.5 stops.

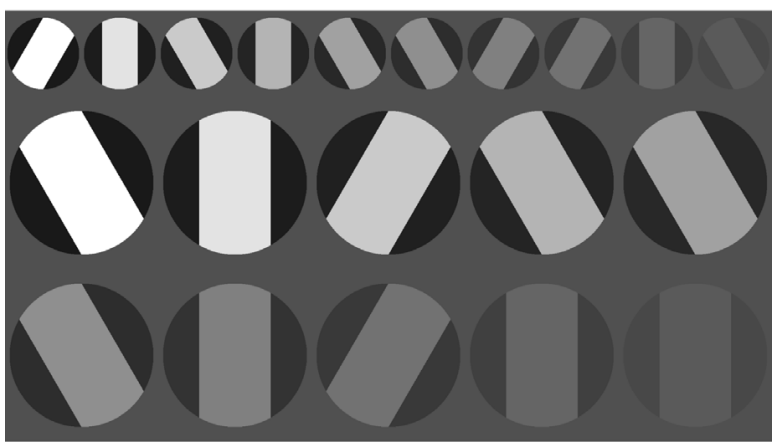

(b) Ayres: circles with a peak luminance of 20 stops above the background.

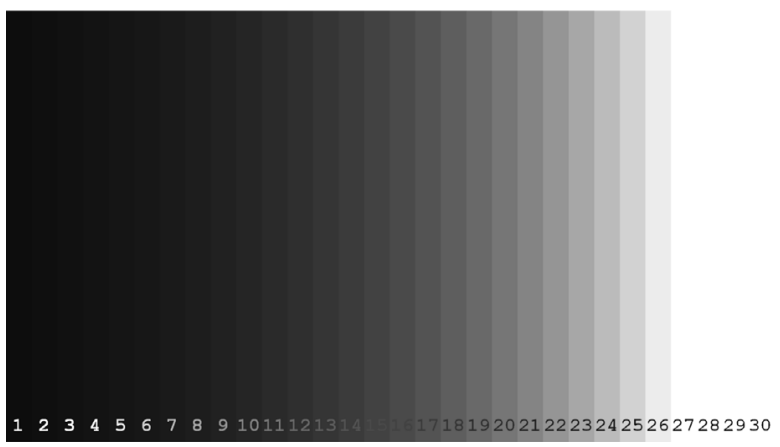

(c) Bars: luminance ramp containing 30 gradations separated by 1 stop each.

Fig. 7. Test charts used in the subjective experiment. The charts were graded at a range of different luminances, 500,1000, 2000, 4000 and 8000 nit. Presented tone-mapped for print. Squares and Ayres show example patterns as the layout was randomised. 
These tasks have been selected as they can be conducted efficiently and provide robust results. In addition, the Ayers chart has been used previously to successfully evaluate the dynamic range of an HDR display [32]. The results of the three tasks under different ambient conditions are the dependent variables of the experiment.

The tasks were performed under two varying conditions corresponding to the two independent variables in the experiment. The first independent variable, brightness, was within-participants and was the luminance of the display. Five conditions were considered: 500, 1000, 2000, 4000 and 8000 nit. All luminance levels were simulated on the same display by adjusting the maximum output brightness.

The second independent variable, ambient, a between-participants independent variable, represents the ambient illumination in the environment. The conditions: 0, 200, 3000 and 80,000 lux were considered. These represent no light, an indoor artificially lit working environment, an indoor sunlit (but not directly) environment, and a sunny outdoor environment respectively. Participants viewed all brightness conditions for a single given ambient condition.

\subsubsection{Materials}

For each of the three dependent variables, a set of test charts were prepared and graded at a range of luminances. The luminances chosen were 8000, 4000, 2000, 1000 and 500 nit, reducing in steps of one stop. A peak luminance of 8000 nit was chosen instead of 10,000 nit as this could be exactly maintained under experimental conditions.

The first chart, shown in Fig. 7a, contained 16 square luminance patches, the brightest patch shown at the peak luminance of the display with the intensity of each successive patch reduced by 1.25 stops to cover a 20 stop range. The patches were displayed on four rows with four patches on each row along with a single black patch; the order of the patches on each row was randomised so as not to allow the prediction of the location of a patch. The visual angle of each square was $2^{\circ} 58^{\prime}$. The goal of the task was to count the number of visible patches.

The second chart, shown in Fig. 7b, contained 20 Ayres circles with varying contrast on a grey background [31], the first row contained 10 smaller circles while the second and third rows contained 5 larger circles. Each was comprised of a dark circle with a light stripe through the middle. Each circle was presented at one of three possible orientations, $-30^{\circ}, 0^{\circ}, 30^{\circ}$, chosen randomly. The circles were displayed over a range of contrasts. The strip in the highest contrast circle was chosen to be the peak luminance of the chart and was 20 stops lighter than the grey background. The dark region was chosen to be 20 stops darker than the background. Each successive circle has a lightness and darkness 2 stops less than the previous giving 20 stops above and below the grey background. The visual angle of the large circles was $2^{\circ} 47^{\prime}$ and the small circles was $1^{\circ} 23^{\prime}$. The goal of the task was to correctly report the orientation of the circle.

The third chart, shown in Fig. 7c, contained 30 full-height bars descending in 1 stop steps from 8000 nit. The chart was clamped at the peak luminance required for the grading. The visual angle of each bar was $0^{\circ} 59^{\prime}$. Each bar was annotated with a number and the goal of the task was to write down the number corresponding to the first and last perceptually differentiable bar allowing the calculation of the number of stops visible.

The luminance of each patch can be calculated by dividing the peak luminance by two raised to the stop value of the patch. The test charts were not tone-mapped prior to display. The display provides a linear relationship between input values and output luminance resulting in a direct correlation between luminance level and information presented. This evaluation is concerned with the correlation between emitted luminance and information perceived.

The specifications for the SIM2 display used in the experiment are shown in Table 2. For the experiment, the display was operated at five different luminance levels: 500, 1000, 2000, 4000 and 8000 nits. The display has an anti-glare coating (haze) of $1 \%$ with a coating
Table 2

Specifications of the display used in the evaluation.

\begin{tabular}{ll}
\hline Parameter & HDR display \\
\hline Brand & SIM2 Multimedia \\
Model & HDR47ES6MB prototype \\
Form factor & $47^{\prime \prime}$ \\
Static contrast ratio & $185,000: 1$ \\
Resolution & $1920 \times 1080$ \\
Viewing angle & $\pm 85^{\circ}$ \\
Peak luminance & $10,000 \mathrm{~cd} / \mathrm{m}^{2}$ \\
\hline
\end{tabular}

Table 3

Measured ambient illuminations in the testing environment.

\begin{tabular}{cl}
\hline Measured ambient illumination (lux) & Time of day \\
\hline 0 & $10 \mathrm{pm}$ \\
500 & $9 \mathrm{pm}$ \\
5000 & $9 \mathrm{am}$ \\
5000 & $6 \mathrm{pm}$ \\
80000 & $12 \mathrm{noon}$ \\
\hline
\end{tabular}

hardness of 3H. A black level of 0.05 nit has been measured for SIM2 displays [33]. It is not possible to measure black levels less than this with a typical Luminance meter, such as the Sekonic L-758D DigitalMaster light meter we used. The darkest stimuli used could not therefore be validated. However, the peak luminance was confirmed with the light meter.

\subsubsection{Experimental environment}

The experiment was performed in two environments, one indoor and one outdoor. The outdoor experiments were conducted at times selected from Table 3 so as to most closely match the target illuminances shown in Table 1 . The actual conditions tested were 0, 200, 3000 and 80,000 lux. To maintain the validity of the results obtained from the experiments, the two first scenarios ( 0 and 200 lux) were conducted in an experimental room in which all the environmental variables were controlled. The remaining conditions were conducted in an outdoor environment with the display in the shadow for the 3000 lux condition and in the sun for the 80,000 lux condition. Care was taken to ensure that the ambient light level did not vary during the time the experiment was conducted. A Sekonic L-758D DigitalMaster was used to measure the environmental illuminances. For the 80,000 lux condition the display was placed at right angles to the sun.

The environments share common features however, the $47^{\prime \prime}$ HDR display was placed approximately $2 \mathrm{~m}$ in-front of the participants who were sitting in groups of five. In each group, the participants were in seated two rows so each has an unencumbered view of the display and were within the required viewing angle of the display to ensure no loss of luminance information.

\subsubsection{Procedure}

Before starting the experiment, the participants were informed that they would be required to perform three tasks: count the number of squares visible on the display; identify the direction of the luminous strip on each circle; report the numbers corresponding to where they could no longer identify the limits between consecutive bars, and were asked to write down the results on paper for later analysis. Then they were introduced to the environment in which their conditions took place and asked to take a seat, ensuring that they can see the entirety of the display. They were also asked to ensure their vision was corrected to normal if required. Each participant was handed a sheet of paper on a clipboard and a pen in order to report their results. The participants were then given five minutes to adapt to the condition. The luminance of the display and illuminance of the environment were recorded right before the start of the experiment. Software then displayed the permutation of the images for the experiment on the display and the participants asked to mark down what they can perceive. Each 
Table 4

Mean scores for the three dependent variables across all brightness and ambient levels. Mean correct answers are shown. Luminance is shown in nits and Illuminance in lux.

\begin{tabular}{|c|c|c|c|c|c|c|}
\hline \multicolumn{7}{|l|}{ (a) Bars } \\
\hline & & \multicolumn{4}{|c|}{ Illuminance } & \multirow[b]{2}{*}{$\mu_{b}$} \\
\hline & & 0 & 200 & 3,000 & 80,000 & \\
\hline \multirow{6}{*}{ Luminance } & 500 & 15.00 & 14.70 & 12.50 & 8.70 & 12.725 \\
\hline & 1,000 & 16.50 & 16.00 & 13.00 & 9.10 & 13.650 \\
\hline & 2,000 & 16.90 & 17.00 & 13.90 & 10.10 & 14.475 \\
\hline & 4,000 & 17.00 & 17.00 & 16.20 & 11.60 & 15.600 \\
\hline & 8,000 & 18.30 & 18.80 & 16.20 & 12.20 & 16.375 \\
\hline & $\mu_{a}$ & 16.74 & 16.82 & 14.36 & 10.34 & \\
\hline
\end{tabular}

\begin{tabular}{|c|c|c|c|c|c|c|}
\hline \multicolumn{7}{|l|}{ (b) Squares } \\
\hline & & \multicolumn{4}{|c|}{ Illuminance } & \multirow[b]{2}{*}{$\mu_{b}$} \\
\hline & & 0 & 200 & 3,000 & 80,000 & \\
\hline \multirow{6}{*}{ Luminance } & 500 & 11.50 & 10.60 & 8.90 & 7.70 & 9.675 \\
\hline & 1,000 & 12.10 & 11.50 & 9.50 & 7.80 & 10.225 \\
\hline & 2,000 & 12.00 & 12.00 & 10.30 & 8.20 & 10.625 \\
\hline & 4,000 & 12.10 & 12.00 & 11.50 & 9.50 & 11.275 \\
\hline & 8,000 & 13.20 & 12.60 & 11.70 & 10.50 & 12.000 \\
\hline & $\mu_{a}$ & 12.18 & 11.74 & 10.38 & 8.75 & \\
\hline
\end{tabular}

\begin{tabular}{|c|c|c|c|c|c|c|}
\hline \multicolumn{7}{|l|}{ (c) Ayres } \\
\hline & & \multicolumn{4}{|c|}{ Illuminance } & \multirow[b]{2}{*}{$\mu_{b}$} \\
\hline & & 0 & 200 & 3,000 & 80,000 & \\
\hline \multirow{6}{*}{ Luminance } & 500 & 17.00 & 16.00 & 12.70 & 9.90 & 13.925 \\
\hline & 1,000 & 18.30 & 16.30 & 14.40 & 11.00 & 15.000 \\
\hline & 2,000 & 18.60 & 18.00 & 15.10 & 11.80 & 15.875 \\
\hline & 4,000 & 19.40 & 18.00 & 15.60 & 12.90 & 16.475 \\
\hline & 8,000 & 20.00 & 19.20 & 16.70 & 13.70 & 17.400 \\
\hline & $\mu_{a}$ & 18.66 & 17.50 & 14.90 & 11.86 & \\
\hline
\end{tabular}

permutation was shown for $15 \mathrm{~s}$, followed by a five second 100 nit grey screen. The total time of the experiment was five minutes. When all the images have been displayed the luminance and illuminance were measured again to ensure they remain consistent.

\subsection{Results}

Descriptive results for each of the three dependent variables are shown in Table 4 to c. Fig. 8 shows the results for all the means. Fig. 9 shows the results in stops averaged across the three dependent variables.

The data captured for bars comprises two values: a minimum and a maximum. For each capture the results are reported as the minimum subtracted from the maximum as this represents the dynamic range observed by the participant. For squares, the number of visible squares are reported. Finally, for Ayres, the number of successful choices is presented.

\subsubsection{Bars results}

For the dependent variable bars, a 4 (ambient) $\times 5$ (brightness) repeated measures factorial ANOVA was conducted.

For the main effect of brightness Mauchly's test of sphericity was violated $(p<0.05)$ and Greenhouse-Geisser corrections were applied. The main effect was significant $F(2.32,83.46)=48.412, p<0.01$. Pairwise comparisons with Bonferroni corrections demonstrated significant differences amongst all the conditions at $p<0.01$ for all conditions. However, 4000 nit against 8000 nit was significant at $p<0.05$.

For the main effect of ambient, a significant difference was also noted $F(3,36)=4117.65, p<0.01$. Pairwise comparisons with Bonferroni corrections found significant differences for all conditions at $p<0.01$ except for the cases of 0 and 200 nit for which no difference was found $p=0.90$.

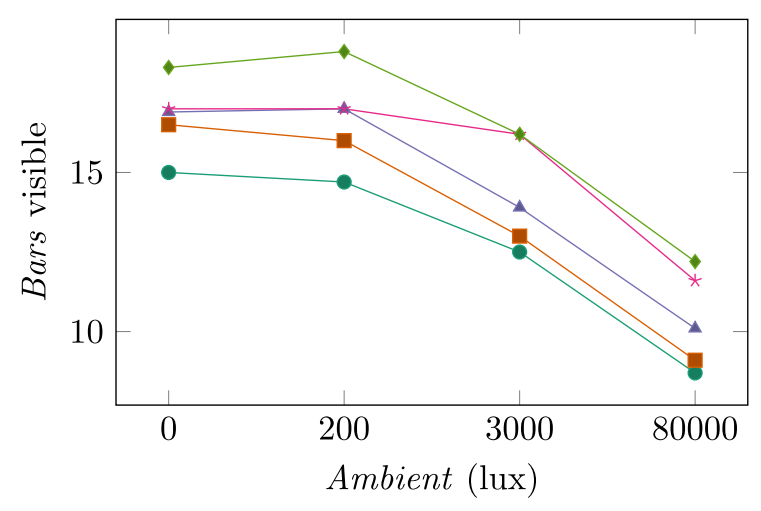

(a) Bars results

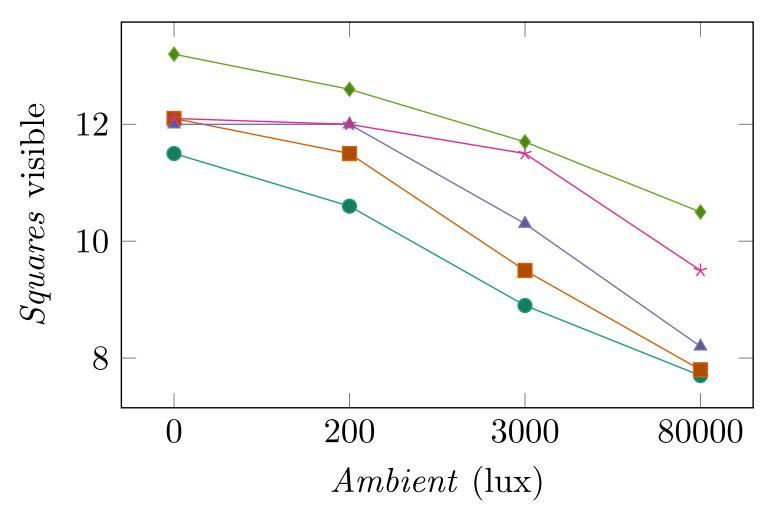

(b) Squares results

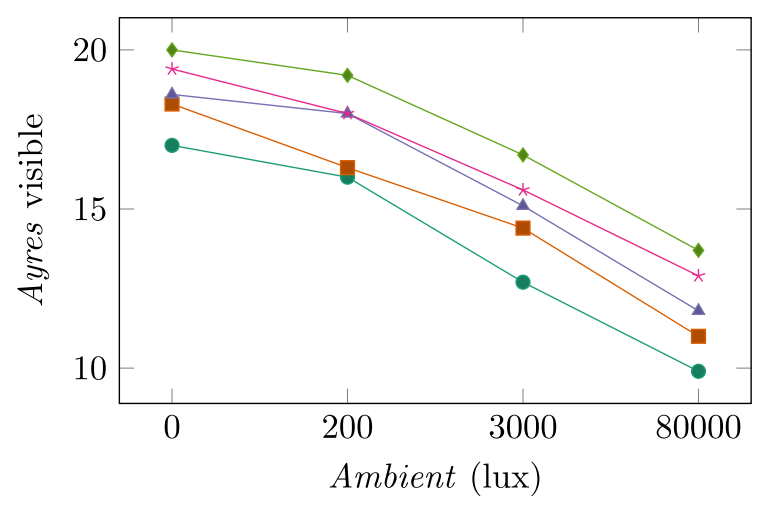

(c) Ayres results

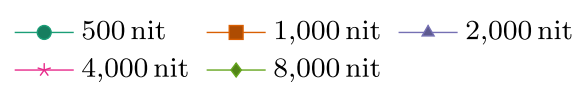

Fig. 8. Mean scores for the three dependent variables.

\subsubsection{Squares results}

The dependent variable squares was also analysed using a 4 (ambient) $\times 5$ (brightness) repeated measures factorial ANOVA.

The main effect of brightness was found to be significant with Greenhouse-Geisser corrections as Mauchly's test for sphericity was violated, $p<0.05, F(2.65,95.56)=63.915, p<0.01$. Pairwise comparisons found significant differences across all brightness conditions.

The main effect of ambient was also found to be significant $F(3,36)$ $=16155.01, p<0.01$. Pairwise comparisons with Bonferroni corrections found significant differences across all conditions except 0 and 200 nit replicating the results seen for bars. 


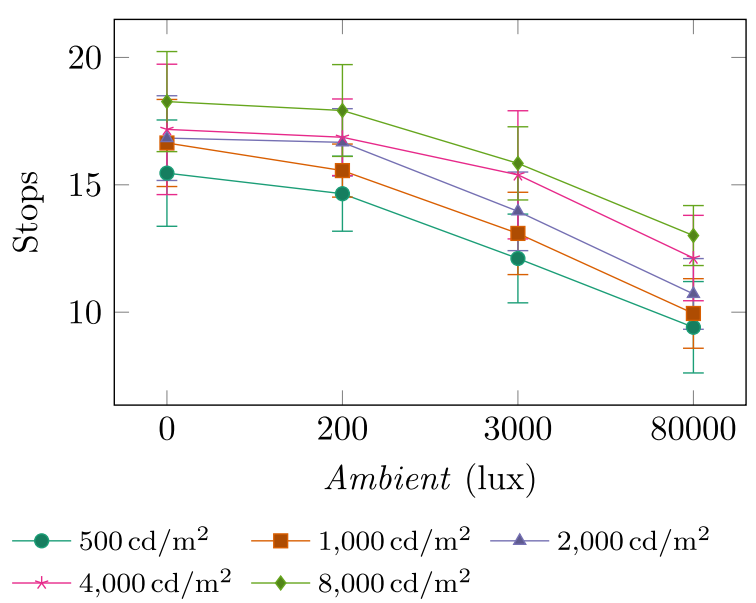

Fig. 9. Mean results in stops for all dependent variables with error bars shown as \pm standard deviation.

\subsubsection{Ayres results}

As with the other dependent variables, Ayres was analysed using 4 (ambient) $\times 5$ (brightness) repeated measures factorial ANOVA. For the main effect of brightness, Greinhouse-Geiser corrections were applied as Mauchly's test of sphericity was violated, $p<0.05$, and a significant effect was found $F(2.86,103.07)=138.47, p<0.01$. Pairwise comparisons with Bonferroni corrections were again found to be significant across all conditions.

The main effect of ambient was found to be significant at $F(3,36)=$ 189.63, $p<0.01$. Pairwise comparisons with Bonferroni corrections found significant differences across all conditions.

\subsection{Analysis}

Our initial hypothesis was that in bright ambient illuminations, a higher peak brightness will allow more information from the image to be perceived and the results show that this is indeed the case. From Fig. 9, the display luminance necessary to perceive a certain range at a given ambient illumination can be estimated, although the results for the darker conditions may depend more on the black level obtained by the display rather than the peak luminance. Around 15.5 stops can be perceived on a 500 nit display in a 200 lux environment, whereas that increases to over 18 stops on an 8000 nit display. In an 80,000 lux environment, the entire range of content containing 10 stops can be perceived correctly on a 1000 nit display, but if the content contains 14 stops an 8000 nit display is needed.

\subsection{Discussion}

The results demonstrate the advantage of utilising a display with a peak luminance of at least 8000 nit. Participants scores for this luminance significantly outperformed the other conditions across all the three dependent variables. It is also clear that the participants' performance at each of the tasks increases as the maximum luminance of the display increases. This is to be expected and confirms our hypothesis.

Another point of note is that as shown in Table 4b, the number of squares that can be seen on a 1000 nit display at 200 lux (11.5) is the same as can be seen on a 4000 nit display at 3000 lux. These results provide an insight into the peak luminance required to match the dynamic range of a scene with the ambient illumination. A system which can automatically adjust to these criteria will ensure the optimal HDR viewing experience while minimising the power required as there is no need to produce a peak luminance higher than the determined value.
The ambient illuminances chosen for this experiment are representative of those in the environments where HDR content may be viewed in the future. It was important to include a sunny environment to show it is possible, with the right peak luminance, to appreciate HDR content outside of a dark environment. Significant differences were found between ambient illuminances confirming the hypothesis that the peak luminance required to display HDR content is related to ambient illumination.

\subsubsection{Limitations}

One limitation with the results is that, because the display is a prototype, although a peak luminance in excess of 10,000 nit was achieved, it proved difficult to maintain a peak luminance level of exactly 10,000 nit for extended periods of time. To ensure consistency for the duration of the subjective evaluation, a maximum peak luminance of 8000 nit was used as this level of luminance could be maintained throughout the evaluation. Furthermore, to give priority to peak luminance, no limiting factor was included on power consumption for the experiment. Despite this limitation, the results clearly show, there is a significant difference between the detail that can been seen with lower peak luminances compared to our proposed display.

Secondly, although the display is nominally rated at a maximum dynamic range of 17.5 stops, participants reported seeing up to 27 stops. The data will therefore need to be tested for robustness before being used further.

\section{Estimating range}

The results of the experiment presented in Section 4 provide an insight into the display luminance required to perceive range in a given ambient illumination. These results can be used to estimate the number of stops visible given the illuminance of the environment and the luminance of the display.

The estimate can be obtained by fitting a surface to the reported data. Locations on this surface can be evaluated and the resulting polynomial expression interpolated to estimate the visible number of stops given the luminance and illuminance.

\subsection{Method}

A linear least-squares fitting method was used to model the participant responses from Section 4 with a $2 \times 1$ degree polynomial surface. The fit was performed on the logarithms of the brightness and ambient variables to linearise perceived response with a small epsilon added to ambient.

No participants failed a test for reliability however nineteen results, shown in Fig. 10, were removed for being greater than $2.7 \sigma(1.5 \times$ the IQR as is standard in box plots [34]) from the condition sample mean.

\subsection{Results \& analysis}

Fig. 11 shows how the surface fits to the participant responses. The adjusted $\mathrm{R}$ square of the fit was 0.7407 . The number of visible stops can be estimated by evaluating the polynomial function:

$f(e, l)=10.072-0.16553 e+0.9017 l-0.060996 e^{2}+0.0435 e l$,

where: $e$ is the log of the ambient +1 and $l$ is the log of the brightness.

Alternatively, the polynomial can be solved for $e$ or $l$. Solving for $e$ allows the estimation of the maximum illuminance in which a display of a given luminance can be used to perceive content with a certain dynamic range. Solving for $l$ allows the estimation of the display luminance required to view content with a certain dynamic range in a given ambient illumination. This is beneficial as the display luminance can be reduced to consume less power if the combination of ambient illuminance and content range does not require the maximum peak brightness for the content to be fully appreciated. 


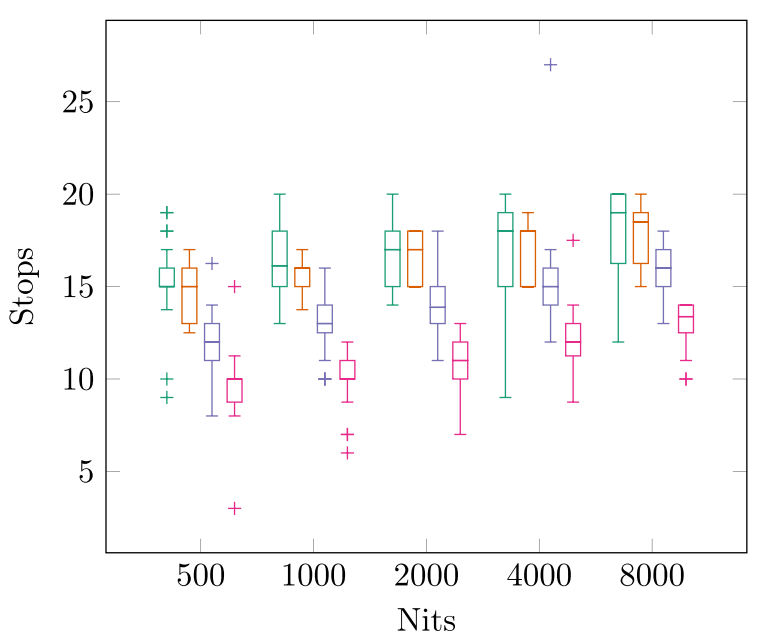

$+0 \operatorname{lux}+200 \operatorname{lux}+3,000 \operatorname{lux}+80,000 \operatorname{lux}$

Fig. 10. Box and whisker plot for each pair of brightness and ambient conditions. The 19 outliers are shown with marks. Less marks are visible as some outlier results are also duplicates.

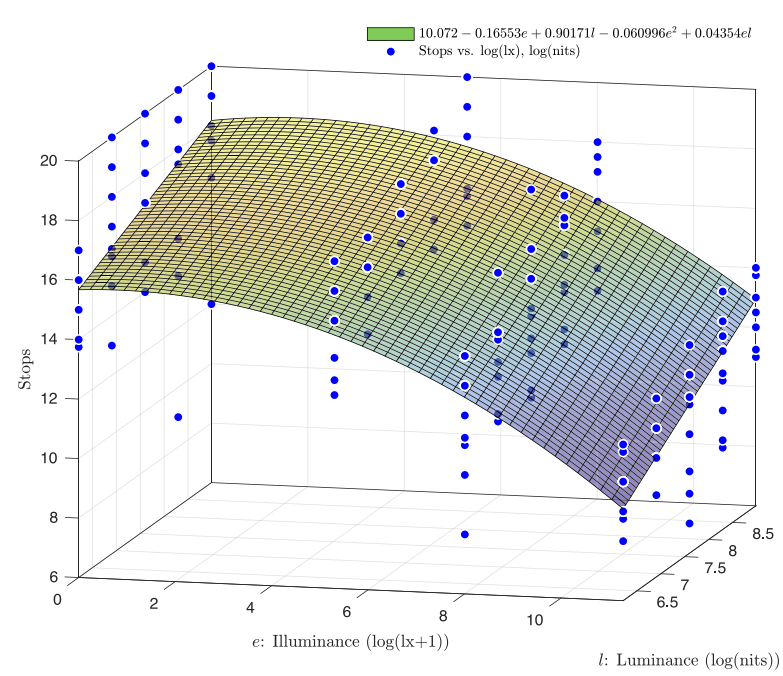

Fig. 11. Surface fit to estimate the visible number of stops from the ambient and brightness.

\section{Conclusions}

The prototype HDR display presented in this paper has shown that it is possible to achieve a 10,000 nit display by innovative use of current LED and LCD technology. With careful thermal management the display does not require liquid cooling, and the overall power consumption can be kept below $1.5 \mathrm{~kW}$ even when running at full peak brightness.

The subjective study has shown that this level of peak brightness is necessary if HDR content is to be seen, and thus enjoyed in bright ambient lighting conditions. Of course, the peak brightness of the display can be automatically reduced if the ambient light level decreases. In particular this can help avoid any problems with eye fatigue due to watching too bright content in a dark environment.

\subsection{Future work}

10,000 nit is the maximum luminance specified for UHD-TV in ITU Recommendation BT.2020 [35]. As LED technology continues to improve, as this work has shown, displays with peak luminances beyond 10,000 nits are possible. This has a number of implications, in particular for UHD compression algorithms. Current methods being considered by, for example SMPTE [36], HDR10 [37] and HLG [38], have been developed for peak luminances of $\leq 10,000$ nit. It is not clear whether these methods will provide the desired quality for displays of more than 10,000 nit and therefore new compression methods may need to be developed to take advantage of future increases in display brightness. Finally, future work will also investigate the impact of eye adaption on viewing experience. Eye adaption is likely to be influenced by the higher dynamic range of the displays as well as brighter ambient lighting conditions.

\section{Acknowledgement}

We are grateful for the EPSRC, UK funding for Jonathan Hatchett's DTG studentship. This work was also partially financed by the ERDF - European Regional Development Fund through the Operational Programme for Competitiveness and Internationalisation - COMPETE 2020 Programme and by National Funds through the Portuguese funding agency, FCT - Fundação para a Ciência e a Tecnologia within project POCI-01-0145-FEDER-028618 entitled PERFECT - Perceptual Equivalence in virtual Reality For authEntiC Training. The experimental study was conducted at INESC TEC's MASSIVE VR Laboratory

\section{References}

[1] F. Banterle, A. Artusi, K. Debattista, A.G. Chalmers, Advanced High Dynamic Range imaging, CRC Press, 2011

[2] Alan Chalmers, Kurt Debattista, HDR Video past, present and future: A perspective, Signal Process., Image Commun. (ISSN: 0923-5965) 54 (2017) 49-55, URL http://www.sciencedirect.com/science/article/pii/S092359651730019X.

[3] Thomas Schulte, J. Barsotti, HDR Demystified: Emerging UHDTV systems, Technical paper, SpecraCal Inc (2016).

[4] Raymond Soneira, 2017 LG OLED TV Display Technology Shoot-Out, URL http: //www.webcitation.org/74auYNrEX.

[5] Gerwin Damberg, James Gregson, Wolfgang Heidrich, High brightness HDR projection using dynamic freeform lensing, ACM Trans. Graph. 35 (3) (2016) 24:1-24:11.

[6] Belen Masia, Gordon Wetzstein, Piotr Didyk, Diego Gutierrez, Special section on advanced displays: A survey on computational displays: Pushing the boundaries of optics, computation, and perception, Comput. Graph. 37 (8) (2013) 1012-1038.

[7] R.R. Hainich, O. Bimber, Displays: Fundamentals and Applications, Taylor \& Francis Inc, 2017.

[8] Adrian Pennington, 10, 000nit display dazzles in the Future Zone, 9 2016. URL http://www.webcitation.org/74yv76ual.

[9] Patrick Ledda, Greg Ward, Alan Chalmers, A wide field, high dynamic range, stereographic viewer, in: GRAPHITE, ACM Press, 2003, pp. 237-244.

[10] ITU-R BT.2246-2: "The present state of ultra-high definition television", 2012 URL http://www.itu.int/dms_pub/itu-r/opb/rep/R-REP-BT.2246-2-2012-PDF-E.pdf.

[11] G. Ward, A wide field, high dynamic range, stereographic viewer, in: SPICS 2002, 2002.

[12] P. Ledda, L.P. Santos, A. Chalmers, A local model of eye adaptation for high dynamic range images, in: Proceedings of the 3rd international conference on Computer graphics, virtual reality, visualisation and interaction in Africa, ACM Press New York, NY, USA, 2004, pp. 151-160.

[13] H. Seetzen, L. Whitehead, G. Ward, A high dynamic range display system using low and high resolution modulators, in: SID Symposium Digest of Technical Papers, Blackwell Publishing Ltd, 2003.

[14] H. Seetzen, W. Heidrich, W. Stuerzlinger, G. Ward, L. Whitehead, M. Trentacoste, A. Ghosh, A. Vorozcovs, High dynamic range display systems, ACM Trans. Graph. 23 (3) (2004).

[15] G. Cutlack, Dolby Develops 4, 000-nit "Pulsar" Television Tech, 122013 URL https://www.webcitation.org/6uyIAXZcw.

[16] J. Ferwerda, S. Luka, A high resolution, high dynamic range display for vision research, J. Vis. 9 (8) (2009).

[17] Cedric Demers, Mehdi Azzabi, 4k vs 1080p and upscaling: Is UHD worth the upgrade?, 62017 URL http://www.webcitation.org/74la7LyeJ.

[18] Cedrick Demers, Peak Brightness of TVs: Max luminosity and HDR highlights, 6 2016 URL http://www.webcitation.org/6yibz80aX.

[19] T. Schulte, HDR Demystified: Emerging UHDTV systems. Technical paper, SpectraCal Inc, 2016.

[20] Vincent Teoh, HDTV test: 4K Blu-ray vs. Blu-ray Reveals HDR Is too dim for daytime, 42016 URL http://www.webcitation.org/6yic6c0rU.

[21] ITU-R BT.709-6: "Parameter Values for the HDTV Standards for Production and International Programme Exchange", 6 2016. URL https://www.itu.int/rec/RREC-BT.709-6-201506-I/en. 
[22] Christopher Moir, Alan Chalmers, The economics of widespread adoption of HDR video, in: High Dynamic Range Video: Concepts, Technologies and Applications, Academic Press, 2016.

[23] Ahmet Oğuz Akyüz, Roland Fleming, Bernhard E. Riecke, Erik Reinhard, Heinrich H. Bülthoff, Do HDR displays support LDR content?: A psychophysical evaluation, ACM Trans. Graph. 26 (3) (2007) http://dx.doi.org/10.1145/ 1276377.1276425.

[24] Ratnajit Mukherjee, Kurt Debattista, Thomas Bashford-Rogers, Brian Waterfield, Alan Chalmers, A study on user preference of high dynamic range over low dynamic range video, Vis. Comput. (ISSN: 1432-2315) 32 (6) (2016) 825-834, http://dx.doi.org/10.1007/s00371-016-1239-7.

[25] Miguel Melo, Maximino Bessa, Luís Barbosa, Kurt Debattista, Alan Chalmers, Screen reflections impact on HDR video tone mapping for mobile devices: an evaluation study, EURASIP Journal on Image and Video Processing (ISSN: 1687-5281) 2015 (1) (2015) 44, http://dx.doi.org/10.1186/s13640-015-0094-1.

[26] Allan G. Rempel, Wolfgang Heidrich, Hiroe Li, Rafał Mantiuk, Video viewing preferences for HDR displays under varying ambient illumination, in: Proceedings of the 6th Symposium on Applied Perception in Graphics and Visualization, in: APGV '09, ACM, New York, NY, USA, ISBN: 978-1-60558-743-1, 2009, pp. 45-52, http://dx.doi.org/10.1145/1620993.1621004.

[27] Scott Daly, Timo Kunkel, Xing Sun, Suzanne Farrell, Poppy Crum, Preference limits of the visual dynamic range for ultra high quality and aesthetic conveyance., in: Human Vision and Electronic Imaging, 3, 2013, p. 86510J.

[28] Scott Daly, Timo Kunkel, Xing Sun, Suzanne Farrell, Poppy Crum, Viewer preferences for shadow, diffuse, specular, and emissive luminance limits of high dynamic range displays, SID Symposium Digest of Technical Papers (ISSN: 2168-0159) 44 (1) (2013) 563-566, http://dx.doi.org/10.1002/j.2168-0159. 2013.tb06271.x, Distinguished Paper.

[29] Hsi-Hao Chung, Sun Lu, Contrast-ratio analysis of sunlight-readable color LCDs for outdoor applications, J. Soc. Inf. Disp. (ISSN: 1938-3657) 11 (1) (2003) 237-242, http://dx.doi.org/10.1889/1.1831713.
[30] ITU-R BT.2022-0 "general viewing conditions for subjective assessment of quality of SDTV and HDTV television pictures on flat panel displays", International Telecommunications Union, Geneva (2012) URL https://www.itu.int/rec/R-RECBT.2022-0-201208-I.

[31] T.J. Ayres, Psychophysical validation of photographic representations, in: The 1996 ASME International Mechanical Engineering Congress and Exposition, 1996, pp. 29-33.

[32] Patrick Ledda, Alan Chalmers, Helge Seetzen, HDR Displays: a validation against reality, in: 2004 IEEE International Conference on Systems, Man and Cybernetics (IEEE Cat. No.04CH37583), Vol. 3, 2004, pp. 2777-2782, http://dx.doi.org/10. 1109/ICSMC.2004.1400753.

[33] Emin Zerman, Giuseppe Valenzise, Francesca De Simone, Francesco Banterle, Frederic Dufaux, Effects of display rendering on HDR image quality assessment, in: SPIE Optical Engineering+ Applications, Applications of Digital Image Processing XXXVIII, San Diego, CA, United States, 2015, URL https://hal.archivesouvertes.fr/hal-01203796.

[34] Boris Iglewicz Michael Frigge, Some implementations of the boxplot, Amer. Statist. 43 (1) (1989) 50-54, http://dx.doi.org/10.1080/00031305.1989. 10475612.

[35] ITU-R BT.2020-2 "Parameter values for ultra-high definition television systems for production and international programme exchange" International Telecommunications Union, Geneva URL https://www.itu.int/rec/R-REC-BT.2020-2201510-I.

[36] SMPTE ST-2084 "High dynamic range electro-optical transfer function of mastering reference displays". 2014.

[37] S. Miller, M. Nezamabadi, S. Daly, Perceptual signal coding for more efficient usage of bit codes, in: SMPTE Conference, 2012.

[38] T. Borer, Non-linear opto-electrical transfer functions for high dynamic range television. White paper, BBC, 2014. 